\title{
Spatial estimates for a class of hyperbolic equations with nonlinear dissipative boundary conditions
}

\author{
Faramarz Tahamtani ${ }^{*}$ and Amir Peyravi
}

* Correspondence: tahamtani@shirazu.ac.ir

Department of Mathematics,

College of Sciences, Shiraz

University, Shiraz, 71454, Iran

\section{Abstract}

This paper is concerned with investigating the spatial behavior of solutions for a class of hyperbolic equations in semi-infinite cylindrical domains, where nonlinear dissipative boundary conditions imposed on the lateral surface of the cylinder. The main tool used is the weighted energy method.

Mathematics Subject Classification (2010) 35B40, 35L05, 35L35

Keywords: Hyperbolic equation, Nonlinear boundary conditions, Phragmén-Lindelöf type theorem, Asymptotic behavior

\section{Introduction}

The aim of this paper is to study the spatial asymptotic behavior of solutions of the problem determined by the equation

$$
u_{t t}=\Delta u_{t}-a u_{t}-\Delta^{2} u, \quad(x, t) \in \Omega \times(0, \infty),
$$

where $a$ is a positive constant and

$$
\Omega=\left\{x \in R^{n}: x_{n} \in R^{+}, x^{\prime}=\left(x_{1}, \ldots, x_{n-1}\right) \in \Gamma_{x_{n}} \subset R^{n-1}\right\},
$$

where

$$
\Gamma_{\tau}=\left\{\left(x^{\prime}, x_{n}\right) \in \Omega: x_{n}=\tau\right\}
$$

When we consider equation (1.1), we impose the initial and boundary conditions

$$
\begin{aligned}
& u(x, 0)=u_{t}(x, 0)=0, \quad x \in \Omega, \\
& u\left(x^{\prime}, 0, t\right)=h_{1}\left(x^{\prime}, t\right), \frac{\partial u}{\partial v}\left(x^{\prime}, 0, t\right)=h_{2}\left(x^{\prime}, t\right), \quad\left(x^{\prime}, t\right) \in \Gamma_{0} \times(0, \infty), \\
& u=0, \Delta u=-f\left(\frac{\partial u}{\partial v}\right), \quad(x, t) \in \Sigma_{0} \times(0, \infty),
\end{aligned}
$$

where $v$ is the outward normal to the boundary and

$$
\Sigma_{\tau}=\left\{x \in R^{n}: x^{\prime} \in \partial \Gamma_{x_{n}}, \tau \leq x_{n}<\infty\right\},
$$


where $\tau \rightarrow \bar{\Gamma}_{\tau}$ is a map from $R^{+}$into family of bounded domains in $R^{n-1}$ with sufficiently smooth boundary $\partial \Gamma_{\tau}$ such that

$$
0<m_{0} \leq \inf _{\tau}\left|\Gamma_{\tau}\right| \leq \sup _{\tau}\left|\Gamma_{\tau}\right| \leq m_{1}<\infty .
$$

In the sequel, we are using

$$
\begin{aligned}
& \Omega_{\tau}=\Omega \cap\left\{x \in R^{n}: 0<x_{n}<\tau\right\}, \\
& R_{\tau}=\Omega \cap\left\{x \in R^{n}: \tau<x_{n}<\infty\right\},
\end{aligned}
$$

and assume $f$ satisfies

$$
\begin{aligned}
& F(v)=\int_{0}^{v} f(\xi) \mathrm{d} \xi \geq \alpha v f(v)>0, \quad \alpha>0, \forall v \in R, \\
& v f(v) \geq \gamma|v|^{2 p}, \quad p>\frac{1}{2}, \gamma>0, \forall v \in R .
\end{aligned}
$$

In recent years, much attention has been directed to the study of spatial behavior of solutions of partial differential equations and systems. The history and development of this question is explained in the work of Horgan and Knowles [1]. The interested reader is referred to the papers [2-9] and the reviews by Horgan and Knowles $[1,10,11]$. The energy method is widely used to study such results.

Spatial growth or decay estimates for nontrivial solutions of initial -boundary value problems in semi-infinite domains with nonlinearities on the boundary have been studied by many authors. Since 1908, when Edvard Phragmén and Ernst Lindelöf published their idea [12], many authors have obtained spatial growth or decay results by Phragmén-Lindelöf theorems. In [13], Horgan and Payne proved some these types of theorems and showed the asymptotic behavior of harmonic functions defined on a three-dimensional semi-infinite cylinder when homogeneous nonlinear boundary conditions are imposed on the lateral surface of the cylinder. Payne and Schaefer [14] proved such results for some classes of heat conduction problems. In [15], Quintanilla investigate the spatial behavior of several nonlinear parabolic equations with nonlinear boundary conditions, (see also $[16,17])$.

Under nonlinear dissipative feedbacks on the boundary, Nouria [18] proved a polynomial stability for regular initial data and exponential stability for some analytic initial data of a square Euler-Bernoulli plate. For the used methodology, one can see [19,20] where the stabilities are investigated in the cases bounded and unbounded feedbacks for some evolution equations. Recently, Celebi and Kalantarov [21] established a Phragmén-Lindelöf type theorems for a linear wave equation under nonlinear boundary conditions. In our study, we establish Phragmén-Lindelöf type theorems for equation (1.1) with nonlinear dissipative feedback terms on the boundary. Our study is inspired by the results of [21].

For the proof of our results, we will use the following Lemma.

Lemma [22]Let $\psi$ be a monotone increasing function with $\psi(0)=0$ and $\lim _{z \rightarrow \infty} \psi(z)=$ $\infty$. Then $\phi(z)>0$ satisfying $\phi(z)<\psi\left(\phi^{\prime}(z)\right), z>0$, tends to $+\infty$ when $z \rightarrow+\infty$. 
(i) If $\psi(z) \leq c z^{m}$ for some $c$ and $m>1$ for $z \geq z_{1}$, then

$$
\liminf _{z \rightarrow+\infty} z^{-\frac{m}{m-1}} \varphi(z)>0 \text {. }
$$

(ii) If $\psi(z) \leq c z$ for some $c$ and $z \geq z_{1}$, then

$$
\liminf _{z \rightarrow+\infty} \varphi(z) \exp \left(-\frac{z}{c}\right)>0 \text {. }
$$

\section{Spatial estimates}

With the solutions of (1.1-1.4) with $h_{i}\left(x^{\prime}, t\right)=0, i=1,2$ is naturally associated an energy function

$$
E(\tau)=\int_{0}^{T}\left[\left\|u_{t}\right\|_{\Omega_{\tau}}^{2}+\left\|\nabla u_{t}\right\|_{\Omega_{\tau}}^{2}+\|\Delta u\|_{\Omega_{\tau}}^{2}+\int_{0}^{\tau} \int_{\partial \Gamma_{\eta}} \nabla u f(\nabla u) \mathrm{d} s \mathrm{~d} \eta\right] \mathrm{d} t,
$$

where $\|.\|_{\Omega}$ denotes the usual norm in $L^{2}(\Omega)$.

A multiplication of equation (1.1) by $u_{t}$, integrating over $\Omega_{\tau}$ and using (1.3-1.5):

$$
\begin{aligned}
& \frac{\mathrm{d}}{\mathrm{d} t}\left[\frac{1}{2}\left\|u_{t}\right\|_{\Omega_{\tau}}^{2}+\frac{1}{2}\|\Delta u\|_{\Omega_{\tau}}^{2}+\int_{0}^{\tau} \int_{\partial \Gamma_{\eta}} F(\nabla u) \mathrm{d} s \mathrm{~d} \eta\right]+a\left\|u_{t}\right\|_{\Omega_{\tau}}^{2} \\
& \quad+\left\|\nabla u_{t}\right\|_{\Omega_{\tau}}^{2}=-\left(u_{t}, u_{x_{n} x_{n} x_{n}}\right)_{\Gamma_{\tau}}+\left(u_{t x_{n}}, u_{x_{n} x_{n}}\right)_{\Gamma_{\tau}}+\left(u_{t}, u_{t x_{n}}\right)_{\Gamma_{\tau}} .
\end{aligned}
$$

Since

$$
\left(u_{t}, u_{x_{n} x_{n} x_{n}}\right)_{\Gamma_{\tau}}=-\left(u_{t x_{n}}, u_{x_{n} x_{n}}\right)_{\Gamma_{\tau}}
$$

we obtain

$$
\begin{gathered}
\frac{\mathrm{d}}{\mathrm{d} t}\left[\frac{1}{2}\left\|u_{t}\right\|_{\Omega_{\tau}}^{2}+\frac{1}{2}\|\Delta u\|_{\Omega_{\tau}}^{2}+\int_{0}^{\tau} \int_{\partial \Gamma_{\eta}} F(\nabla u) \mathrm{d} s \mathrm{~d} \eta\right]+a\left\|u_{t}\right\|_{\Omega_{\tau}}^{2} \\
\quad+\left\|\nabla u_{t}\right\|_{\Omega_{\tau}}^{2}=2\left(u_{t x_{n}}, u_{x_{n} x_{n}}\right)_{\Gamma_{\tau}}+\left(u_{t}, u_{t x_{n}}\right)_{\Gamma_{\tau}} .
\end{gathered}
$$

Let $\delta>0$. Multiplying (1.1) by $\delta u$, integrating over $\Omega_{\tau}$, and adding to (2.2), we obtain

$$
\begin{aligned}
& \frac{\mathrm{d}}{\mathrm{d} t}\left\{\frac{1}{2}\left\|u_{t}\right\|_{\Omega_{\tau}}^{2}+\frac{1}{2}\|\Delta u\|_{\Omega_{\tau}}^{2}+\delta\left(u, u_{t}\right)_{\Omega_{\tau}}\right. \\
& \left.\quad+\frac{a \delta}{2}\|u\|_{\Omega_{\tau}}^{2}+\frac{\delta}{2}\|\nabla u\|_{\Omega_{\tau}}^{2}+\int_{0}^{\tau} \int_{\partial \Gamma_{\eta}} F(\nabla u) \mathrm{d} s \mathrm{~d} \eta\right\} \\
& \quad+(a-\delta)\left\|u_{t}\right\|_{\Omega_{\tau}}^{2}+\left\|\nabla u_{t}\right\|_{\Omega_{\tau}}^{2}+\delta\|\Delta u\|_{\Omega_{\tau}}^{2}+\delta \int_{0}^{\tau} \int_{\partial \Gamma_{\eta}} \nabla u f(\nabla u) \mathrm{d} s \mathrm{~d} \eta \\
& \quad=2\left(u_{t x_{n}}, u_{x_{n} x_{n}}\right)_{\Gamma_{\tau}}+\left(u_{t}, u_{t x_{n}}\right)_{\Gamma_{\tau}}+2 \delta\left(u_{x_{n}}, u_{x_{n} x_{n}}\right)_{\Gamma_{\tau}}+\delta\left(u, u_{t x_{n}}\right)_{\Gamma_{\tau}} .
\end{aligned}
$$


Integrating (2.3) with respect to $t$ over $(0, T)$ and using (1.5), one can find

$$
\begin{aligned}
& \frac{1}{2}\left\|u_{t}\right\|_{\Omega_{\tau}}^{2}+\frac{1}{2}\|\Delta u\|_{\Omega_{\tau}}^{2}+\frac{\delta}{2}\|\nabla u\|_{\Omega_{\tau}}^{2}+\frac{a \delta}{2}\|u\|_{\Omega_{\tau}}^{2} \\
& \quad+\delta\left(u, u_{t}\right)_{\Omega_{\tau}}+\alpha \int_{0}^{\tau} \int_{\partial \Gamma \eta} \nabla u f(\nabla u) \mathrm{d} s \mathrm{~d} \eta \\
& \quad+(a-\delta) \int_{0}^{T}\left\|u_{t}\right\|_{\Omega_{\tau}}^{2} \mathrm{~d} t+\delta \int_{0}^{T}\|\Delta u\|_{\Omega_{\tau}}^{2} \mathrm{~d} t+\int_{0}^{T}\left\|\nabla u_{t}\right\|_{\Omega_{\tau}}^{2} \mathrm{~d} t \\
& +\delta \int_{0}^{T} \int_{0}^{\tau} \int_{\partial \Gamma \eta} \nabla u f(\nabla u) \mathrm{d} s \mathrm{~d} \eta d t \leq \int_{0}^{T}\left[2\left(u_{t x_{n}}, u_{x_{n} x_{n}}\right) \Gamma_{\tau}+\left(u_{t}, u_{t x_{n}}\right) \Gamma_{\tau}\right] \mathrm{d} t \\
& \quad+\int_{0}^{T}\left[2 \delta\left(u_{x_{n}}, u_{x_{n} x_{n}}\right) \Gamma_{\tau}+\delta\left(u, u_{t x_{n}}\right) \Gamma_{\tau}\right] \mathrm{d} t .
\end{aligned}
$$

On exploiting (2.1) and the inequality $-\left(\frac{1}{4}\right)\left\|u_{t}\right\|_{\Omega_{\tau}}^{2}-\delta^{2}\|u\|_{\Omega_{\tau}}^{2} \leq \delta\left(u, u_{t}\right)_{\Omega_{\tau}}$, the estimate (2.4) takes the form

$$
\begin{aligned}
\sigma^{-1} E(\tau) \leq & \int_{0}^{T}\left[\left(u_{t x_{n}}, u_{x_{n} x_{n}}\right) \Gamma_{\tau}+\left(u_{t}, u_{t x_{n}}\right) \Gamma_{\tau}\right] \mathrm{d} t \\
& +\int_{0}^{T}\left[\left(u_{x_{n}}, u_{x_{n} x_{n}}\right) \Gamma_{\tau}+\left(u, u_{t x_{n}}\right) \Gamma_{\tau}\right] \mathrm{d} t
\end{aligned}
$$

by choosing $\delta=\frac{a}{2}, \delta_{1}=\min \left\{1, \frac{a}{2}\right\}, \sigma=\max \left\{\frac{a}{\delta_{1}}, \frac{2}{\delta_{1}}\right\}$. Now we find upper bounds for the right hand side of (2.5). Using the Young's and Schwartz inequalities, we have

$$
\begin{aligned}
& \int_{0}^{T}\left(u_{t x_{n}}, u_{x_{n} x_{n}}\right)_{\Gamma_{\tau}} \mathrm{d} t \leq \frac{1}{2} \int_{0}^{T}\left\|\nabla u_{t}\right\|_{\Gamma_{\tau}}^{2} \mathrm{~d} t+\frac{1}{2} \int_{0}^{T}\|\Delta u\|_{\Gamma_{\tau}}^{2} \mathrm{~d} t \\
& \int_{0}^{T}\left(u_{t}, u_{t x_{n}}\right)_{\Gamma_{\tau}} \mathrm{d} t \leq \frac{1}{2} \int_{0}^{T}\left\|u_{t}\right\|_{\Gamma_{\tau}}^{2} \mathrm{~d} t+\frac{1}{2} \int_{0}^{T}\left\|\nabla u_{t}\right\|_{\Gamma_{\tau}}^{2} \mathrm{~d} t \\
& \int_{0}^{T}\left(u_{x_{n}}, u_{x_{n} x_{n}}\right)_{\Gamma_{\tau}} \mathrm{d} t \leq\left.\int_{0}^{T}\left\|u_{x_{n}}\right\|_{\Gamma_{\tau}}\left\|u_{x_{n} x_{n}}\right\|\right|_{\Gamma_{\tau}} \mathrm{d} t .
\end{aligned}
$$

By the Poincaré inequality, it is not difficult to see

$$
\|v\|_{D}^{2} \leq \lambda^{-1}\|\nabla v\|_{D}^{2}+|D|^{-1}\left(\int_{D} v \mathrm{~d} A\right)^{2} .
$$


Inserting (2.9) into (2.8), we get

$$
\begin{aligned}
& \int_{0}^{T}\left(u_{x_{n}}, u_{x_{n} x_{n}}\right)_{\Gamma_{\tau}} \mathrm{d} t \\
& \leq \int_{0}^{T}\left\{\lambda_{\tau}^{-\frac{1}{2}}|| \Delta^{\prime} u \|_{\Gamma_{\tau}}+\left|\Gamma_{\tau}\right|^{-\frac{1}{2}}\left|\int_{\Gamma_{\tau}} \nabla^{\prime} u \mathrm{~d} A\right|\right\}|| u_{x_{n} x_{n}} \|_{\Gamma_{\tau}} \mathrm{d} t,
\end{aligned}
$$

where $\Delta^{\prime}$ and $\nabla^{\prime}$ are Laplacian and gradient operators in $R^{n-1}$, respectively, $\left|\Gamma_{\tau}\right|$ is the area of $\Gamma_{\tau}$ and $\lambda_{\tau}$ is the Poincaré constant. Now, we recall the inequality

$$
\int_{D} v \mathrm{~d} A \leq \frac{r_{0}}{2} \int_{\partial D}|v| \mathrm{d} s+\frac{I_{0}^{\frac{1}{2}}}{2}\left(\int_{D}|\nabla v|^{2} \mathrm{~d} A\right)^{\frac{1}{2}}
$$

from [13] where $r_{0}^{2}=\sup _{D}\left|x^{\prime}\right|^{2}$ and $I_{0}=\int_{D}\left|x^{\prime}\right|^{2} \mathrm{~d} A$. Using (2.11) and the Hölder's inequality to estimate the boundary integral $\left|\int_{\Gamma_{\tau}} \nabla^{\prime} u \mathrm{~d} A\right|$ in (2.10), we obtain

$$
\begin{aligned}
& \int_{0}^{T}\left(u_{x_{n}}, u_{x_{n} x_{n}}\right)_{\Gamma_{\tau}} \mathrm{d} t \\
& \quad \leq \int_{0}^{T}\left\{M_{1}\left\|\Delta^{\prime} u\right\|_{\Gamma_{\tau}}+\gamma^{\frac{1}{2 p}} M_{2}\left(\int_{\partial \Gamma_{\tau}}\left|\nabla^{\prime} u\right|^{2 p} \mathrm{~d} A\right)^{\frac{1}{2 p}}\right\}\left\|u_{x_{n} x_{n}}\right\|_{\Gamma_{\tau}} \mathrm{d} t
\end{aligned}
$$

where $M_{1}=\lambda^{-\frac{1}{2}}+\frac{I^{1 / 2}}{2 m^{1 / 2}}, M_{2}=\frac{1}{2} r L^{(2 p-1) / 2 p} m^{-1 / 2} \gamma^{-1 / 2 p}$, such that $r=\sup _{\tau} r_{\tau}, \lambda=\inf _{\tau}$ $\lambda_{\tau}, I=\sup _{\tau} I_{\tau}, L=\sup _{\tau} L_{\tau}$ and $m=\inf _{\tau}\left|\Gamma_{\tau}\right|$ in which $L_{\tau}$ is the area of $\partial \Gamma_{\tau}$. From (1.6) the inequality (2.12) yields

$$
\begin{aligned}
& \int_{0}^{T}\left(u_{x_{n}}, u_{x_{n} x_{n}}\right)_{\Gamma_{\tau}} \mathrm{d} t \\
& \leq M_{1} \int_{0}^{T}\|\Delta u\|_{\Gamma_{\tau}}^{2} \mathrm{~d} t+M_{2} \int_{0}^{T}\left(\int_{\Gamma_{\tau}} \nabla^{\prime} u f\left(\nabla^{\prime} u\right) \mathrm{d} A\right)^{\frac{1}{2 p}}\left\|u_{x_{n} x_{n}}\right\|_{\Gamma_{\tau}} \mathrm{d} t .
\end{aligned}
$$


Consequently

$$
\begin{gathered}
\left(\int_{\partial \Gamma_{\tau}} \nabla^{\prime} u f\left(\nabla^{\prime} u\right) \mathrm{d} s\right)^{\frac{1}{2 p}}\left(\int_{\Gamma_{\tau}} u_{x_{n} x_{n}}^{2} \mathrm{~d} A\right)^{\frac{1}{2}} \\
=\left[\left(\int_{\partial \Gamma_{\tau}} \nabla^{\prime} u f\left(\nabla^{\prime} u\right) \mathrm{d} s\right)^{\frac{1}{p+1}}\left(\int_{\Gamma_{\tau}} u_{x_{n} x_{n}}^{2} \mathrm{~d} A\right)^{\frac{p}{p+1}}\right]^{\frac{p+1}{2 p}} \\
\leq\left[\frac{\mu^{p}}{1+p} \int_{\partial \Gamma_{\tau}} \nabla^{\prime} u f\left(\nabla^{\prime} u\right) \mathrm{d} s+\frac{p}{\mu(1+p)} \int_{\Gamma_{\tau}} u_{x_{n} x_{n}}^{2} \mathrm{~d} A\right]^{\frac{p+1}{2 p}},
\end{gathered}
$$

where the Young's inequality

$$
\alpha^{\varepsilon} \beta^{1-\varepsilon}=(\alpha \gamma)^{\varepsilon}\left[\beta \gamma^{\frac{-\varepsilon}{1-\varepsilon}}\right]^{(1-\varepsilon)} \leq \varepsilon \alpha \gamma+(1-\varepsilon) \beta \gamma \frac{-\varepsilon}{1-\varepsilon},
$$

for $0<\varepsilon<1, \mu=p^{\frac{1}{p+1}}$ and $\gamma=\mu^{p}$ have been used. Therefore,

$$
\begin{aligned}
& \left(\int_{\Gamma_{\tau}} \nabla^{\prime} u f\left(\nabla^{\prime} u\right) \mathrm{d} s\right)^{\frac{1}{2 p}}\left(\int_{\Gamma_{\tau}} u_{x_{n} x_{n}}^{2} \mathrm{~d} A\right)^{\frac{1}{2}} \\
& \leq\left[N(p)\left(\int_{\partial \Gamma_{\tau}} \nabla^{\prime} u f\left(\nabla^{\prime} u\right) \mathrm{d} s+\int_{\Gamma_{\tau}} u_{x_{n} x_{n}}^{2} \mathrm{~d} A\right)\right]^{\frac{p+1}{2 p}},
\end{aligned}
$$

where

$$
N(p)=\frac{p^{\frac{p}{p+1}}}{(1+p)}
$$

By using (2.13) and (2.14), we get

$$
\begin{aligned}
& \int_{0}^{T}\left(u_{x_{n}}, u_{x_{n} x_{n}}\right)_{\Gamma_{\tau}} \mathrm{d} t \leq M_{1} \int_{0}^{T}\|\Delta u\|_{\Gamma_{\tau}}^{2} \mathrm{~d} t \\
& +M_{2} \tilde{N}(p) \int_{0}^{T}\left(\int_{\partial \Gamma_{\tau}} \nabla^{\prime} u f\left(\nabla^{\prime} u\right) \mathrm{d} s+\int_{\Gamma_{\tau}} u_{x_{n} x_{n}}^{2} \mathrm{~d} A\right)^{\frac{p+1}{2 p}} \mathrm{~d} t
\end{aligned}
$$


where $\tilde{N}(p)=[N(p)]^{\frac{p+1}{2 p}}$. From (2.15), it is easy to see

$$
\begin{aligned}
& \int_{0}^{T}\left(u_{x_{n}}, u_{x_{n} x_{n}}\right)_{\Gamma_{\tau}} \mathrm{d} t \leq M_{1} \int_{0}^{T}\|\Delta u\|_{\Gamma_{\tau}}^{2} \mathrm{~d} t \\
& +M_{2} C \tilde{N}(p)\left(\int_{0}^{T} \int_{\partial \Gamma_{\tau}} \nabla u f(\nabla u) \mathrm{d} s \mathrm{~d} t+\int_{0}^{T}\|\Delta u\|_{\Gamma_{\tau}}^{2} \mathrm{~d} t\right)^{\frac{p+1}{2 p}},
\end{aligned}
$$

where $C$ is a positive constant.

Next, we exploit Poincaré inequality to estimate

$$
\int_{0}^{T}\left(u, u_{t x_{n}}\right)_{\Gamma_{\tau}} \mathrm{d} t \leq \frac{\rho^{-1}}{2} \int_{0}^{T}\|\Delta u\|_{\Gamma_{\tau}}^{2} \mathrm{~d} t+\frac{1}{2} \int_{0}^{T}\left\|\nabla u_{t}\right\|_{\Gamma_{\tau}}^{2} \mathrm{~d} t,
$$

where $\rho$ is the Poincaré constant.

Now, from the inequalities (2.5-2.7), (2.16), and (2.17), one can find

$$
\begin{aligned}
& E(\tau) \leq \int_{0}^{T}\left[\frac{\sigma}{2}\left\|u_{t}\right\|_{\Gamma_{\tau}}^{2}+\frac{3}{2} \sigma\left\|\nabla u_{t}\right\|_{\Gamma_{\tau}}^{2}+\sigma\left(\frac{1}{2}+M_{1}+\frac{\rho^{-1}}{2}\right)\|\Delta u\|_{\Gamma_{\tau}}^{2}\right] \mathrm{d} t \\
& +\int_{0}^{T} \int_{\partial \Gamma_{\tau}} \nabla u f(\nabla u) \mathrm{d} s \mathrm{~d} t+\sigma M_{2} C \tilde{N}(p)\left\{\int_{0}^{T} \int_{\partial \Gamma_{\tau}} \nabla u f(\nabla u) \mathrm{d} s \mathrm{~d} t\right. \\
& \left.+\int_{0}^{T}\left[\left\|\nabla u_{t}\right\|_{\Gamma_{\tau}}^{2} \mathrm{~d} t+\|\Delta u\|_{\Gamma_{\tau}}^{2}+\left\|u_{t}\right\|_{\Gamma_{\tau}}^{2}\right] \mathrm{d} t\right\}^{\frac{p+1}{2 p}} .
\end{aligned}
$$

Upon inserting (2.1) into the right hand side of (2.18), we may write an inequality in the form

$$
E(\tau) \leq \sigma\left(\frac{5}{2}+M_{1}+\frac{\rho^{-1}}{2}\right) E^{\prime}(\tau)+\sigma M_{2} C \tilde{N}(p)\left[E^{\prime}(\tau)\right]^{\frac{p+1}{2 p}} .
$$

At this point, by the inequality (2.19), the function $\psi(z)=\alpha_{1} z+\alpha_{2} z^{\frac{p+1}{2 p}}$ satisfies in the hypothesis of the Lemma. Therefore, we have proved the following theorem.

Theorem 1 Let $u(x, t)$ be a nontrivial solution of (1.1) - (1.4) with $h_{i}\left(x^{\prime}, t\right)=0, i=1$, 2 under the conditions (1.5) and (1.6). Then

$$
\liminf _{\tau \rightarrow+\infty} E(\tau) \tau^{-\frac{p+1}{1-p}}>0, \quad p \in\left(\frac{1}{2}, 1\right),
$$

and

$$
\liminf _{\tau \rightarrow+\infty} E(\tau) \exp \left(-\frac{\tau}{c}\right)>0, \quad p \in[1,+\infty),
$$

where

$$
c=\max \left\{\sigma\left(\frac{5}{2}+M_{1}+\frac{\rho^{-1}}{2}\right), \sigma M_{2} C \tilde{N}(p)\right\} .
$$


Theorem 2 Consider the equation (1.1) subject to the conditions $u\left(x^{\prime}, 0, t\right)=h_{1}\left(x^{\prime}, t\right)$ and $\frac{\partial u}{\partial v}\left(x^{\prime}, 0, t\right)=h_{2}\left(x^{\prime}, t\right)$ for $x^{\prime} \in \Gamma_{0}$. If $E(+\infty)$ is finite, then

$$
\lim _{\tau \rightarrow+\infty}\left(\int_{0}^{T}\left\|u_{t}\right\|_{R_{\tau}}^{2} \mathrm{~d} t+\int_{0}^{T}\left\|\nabla u_{t}\right\|_{R_{\tau}}^{2} \mathrm{~d} t+\int_{0}^{T}\|\Delta u\|_{R_{\tau}}^{2} \mathrm{~d} t\right)=0 .
$$

proof By the same manner followed in theorem 1, it is easy to find the inequality

$$
\begin{gathered}
(a-\delta) \int_{0}^{T}\left\|u_{t}\right\|_{R_{\tau}}^{2} \mathrm{~d} t+\int_{0}^{T}\left\|\nabla u_{t}\right\|_{R_{\tau}}^{2} \mathrm{~d} t+\delta \int_{0}^{T}\|\Delta u\|_{R_{\tau}}^{2} \mathrm{~d} t \leq \frac{1}{2} \int_{0}^{T}\left\|u_{t}\right\|_{\Gamma_{\tau}}^{2} \mathrm{~d} t \\
+\left(\frac{3}{2}+\frac{\delta}{2}\right) \int_{0}^{T}\left\|\nabla u_{t}\right\|_{\Gamma_{\tau}}^{2} \mathrm{~d} t+\left[1+\delta\left(1+\lambda_{\tau}^{-1}+\frac{1}{2} \lambda_{\tau}^{-2}\right)\right] \int_{0}^{T}\|\Delta u\|_{\Gamma_{\tau}}^{2} \mathrm{~d} t,
\end{gathered}
$$

where $\lambda_{\tau}$ is the Poincaré constant. Choosing $\delta \in(0, a), \eta=\min \{a-\delta, \delta, 1\}$ and

$$
\tilde{\gamma}=\eta^{-1} \max \left\{\frac{3}{2}+\frac{\delta}{2}, 1+\delta\left(1+\lambda_{\tau}^{-1}+\frac{1}{2} \lambda_{\tau}^{-2}\right)\right\},
$$

we obtain

$$
\tilde{E}(\tau) \leq-\tilde{\gamma} \tilde{E}^{\prime}(\tau)
$$

where

$$
\tilde{E}(\tau)=\int_{0}^{T}\left\|u_{t}\right\|_{R_{\tau}}^{2} \mathrm{~d} t+\int_{0}^{T}\left\|\nabla u_{t}\right\|_{R_{\tau}}^{2} \mathrm{~d} t+\int_{0}^{T}\|\Delta u\|_{R_{\tau}}^{2} \mathrm{~d} t .
$$

Thus, (2.20) follows from (2.21).

\section{Authors' contributions}

The authors declare that the work was realized in collaboration with the same responsibility. All authors read and approved the final manuscript.

\section{Competing interests}

The authors declare that they have no competing interests.

Received: 3 April 2011 Accepted: 30 August 2011 Published: 30 August 2011

\section{References}

1. Horgan, CO, Knowles, JK: Recent developments concerning Saint-Venant's principle. In: Wu TY, Hutchinson JW (eds.) Advances in Applied Mechanics, vol. 23, pp. 179-269. Academic Press, New York (1983)

2. Celebi, AO, Kalantarov, VK, Tahamtani, F: Phragmén-Lindelöf type theorems for some semilinear elliptic and parabolic equations. Demonstratio Mathematica. 31, 43-54 (1998)

3. Flavin, JN: On Knowles'version of Saint-Venant's principle in two-dimensional elastostatics. Arch Ration Mech Anal. 53 , 366-375 (1974). doi:10.1007/BF00281492

4. Flavin, JN, Knops, RJ, Payne, LE: Decay Estimates for the Constrained Elastic Cylinder of Variable Cross Section. Quart Appl Math. XLVII, 325-350 (1989)

5. Flavin, JN, Knops, RJ: Asymptotic behaviour of solutions to semi-linear elliptic equations on the half cylinder. Z Angew Math phys. 43, 405-421 (1992). doi:10.1007/BF00946237

6. Flavin, JN, Rionero, S: Qualitative Estimates for Partial Differential Equations, An Introduction. CRC Press, Roca Raton (1996)

7. Horgan, CO: Decay estimates for the biharmonic equation with applications to Saint-Venant principles in plane elasticity and Stokes flow. Quart Appl Math. 47, 147-157 (1989)

8. Knowles, JK: On Saint-Venant's principle in the two-dimensional linear theory of elasticity. Arch Ration Mech Anal. 21, 123-144 (1966)

9. Knowles, JK: An energy estimate for the biharmonic equation and its application to Saint-Venant's principle in plane elastostatics. Indian J Pure Appl Math. 14, 791-805 (1983) 
10. Horgan, CO: Recent developments concerning Saint-Venant's principle: An update. Appl Mech Rev. 42, 295-303 (1989). doi:10.1115/1.3152414

11. Horgan, CO: Recent developments concerning Saint-Venant's principle: A second update. Appl Mech Rev. 49, s101-s111 (1996). doi:10.1115/1.3101961

12. Phragmén, $\mathrm{E}$, Lindelöf, E: Sur une extension d'un principle classique de l'analyse et sur quelque propriétès des functions monogènes dans le voisinage d'un point singulier. Acta Math. 31, 381-406 (1908). doi:10.1007/BF02415450

13. Horgan, CO, Payne, LE: Phragmén-Lindelöf Type Results for Harmonic Functions with Nonlinear Boundary Conditions. Arch Rational Mech Anal. 122, 123-144 (1993). doi:10.1007/BF00378164

14. Payne, LE, Schaefer, PW, Song, JC: Growth and decay results in heat conduction problems with nonlinear boundary conditions. Nonlinear Anal. 35, 269-286 (1999). doi:10.1016/50362-546X(98)00034-0

15. Quintanilla, R: On the spatial blow-up and decay for some nonlinear boundary conditions. Z angew Math Phys. 57, 595-603 (2006). doi:10.1007/s00033-005-0035-4

16. Quintanilla, R: Comparison arguments and decay estimates in nonlinear viscoelasticity. Int J Non-linear Mech. 39, 55-61 (2004). doi:10.1016/S0020-7462(02)00127-0

17. Quintanilla, R: Phragmén-Lindelöf alternative for the displacement boundary value problem in a theory of nonlinear micropolar elasticity. Int J Non-linear Mech. 41, 844-849 (2006). doi:10.1016/j.jinonlinmec.2006.06.001

18. Nouria, S: Polynomial and analytic boundary feedback stabilization of square plate. Bol Soc Parana Math. 27(2):23-43 (2009)

19. Ammari, K, Tucsnak, M: Stabilization of second order evolution equations by a class of unbounded feedbacks, ESIM: Control Optim. Calc Var. 6, 361-386 (2001)

20. Haraux, A: Series lacunaires et controle semi-interene des vibrations d'une plaque rectangulaire. J Math Pures App. 68, 457-465 (1989)

21. Celebi, AO, Kalantarov, VK: Spatial behaviour estimates for the wave equation under nonlinear boundary condition. Math Comp Model. 34, 527-532 (2001). doi:10.1016/50895-7177(01)00080-2

22. Ladyzhenskaya, OA, Solonnikov, VA: Determination of solutions of boundary value problems for stationary Stokes and Navier-Stokes equations having an unbounded Dirichlet integral. Zap Nauch Semin LOMI. 96, 117-160 (1980)

doi:10.1186/1687-2770-2011-19

Cite this article as: Tahamtani and Peyravi: Spatial estimates for a class of hyperbolic equations with nonlinear dissipative boundary conditions. Boundary Value Problems 2011 2011:19.

\section{Submit your manuscript to a SpringerOpen ${ }^{\odot}$} journal and benefit from:

- Convenient online submission

- Rigorous peer review

- Immediate publication on acceptance

- Open access: articles freely available online

- High visibility within the field

- Retaining the copyright to your article

Submit your next manuscript at $\gg$ springeropen.com 